\title{
MODERNIZACIÓN Y TRANSFORMACIONES DE LAS FAMILIAS COMO PROCESOS DEL CONDICIONAMIENTO SOCIAL DE DOS GENERACIONES*
}

\author{
EUGENIO GUTIÉRREZ ${ }^{* *}$ \\ PAULINA OSORIO $^{* * *}$
}

\begin{abstract}
RESUMEN
El presente artículo entrega una revisión de la modernización de la sociedad chilena en relación a sus efectos en la familia, con la intención de contribuir en la identificación de aspectos que participan en el proceso de condicionamiento de dos generaciones del siglo XX. Esta aproximación se realizó poniendo especial atención al papel del Estado y las relaciones de pareja y entre padres e hijos.
\end{abstract}

PALABRAS ClAVE: ESTADO, EDADES, GÉNERO

* Este texto se inscribe en el marco de ejecución del proyecto ConicytBanco Mundial, Anillos en Ciencias Sociales (ACS-33), «Observatorio Social del Envejecimiento y la Vejez en Chile».

** Antropólogo. Investigador de la Corporación Años, Santiago, Chile. E-mail: eugeniogutierrezv@mi.cl.

*** Antropóloga Social y Doctora en Sociología. Académica de la Universidad de Chile, Santiago, Chile; e investigadora de la Corporación Años, Santiago, Chile. E-Mail: posorio@uchile.cl. 


\title{
RESUMO
}

O presente artigo analisa os efeitos da modernização da sociedade chilena sobre a família, com o objetivo de contribuir na identificação de aspectos presentes no processo de condicionamento de duas gerações do século XX. Realizou-se esta aproximação dando-se atenção especial ao papel do Estado, às relações de casal e entre pais e filhos.

PALAVRAS CHAVE: ESTADO, IDADES, GÊNERO

\begin{abstract}
The present article gives a revision of the modernization of the Chilean society in relation to its effects in the family, with the intention to contribute to the identification of aspects that participate in the process of the agreement of two generations of the $20^{\text {th }}$ Centuary. This approach was realised by putting special attention to the role played by the State and the relationships of couples and between parents and children.
\end{abstract}

KEY WORDS: STATE, AGES, GENERIC 


\section{INTRODUCCIÓN}

LOS ESCENARIOS QUE SE describen a continuación como condicionantes de dos generaciones, corresponden a dos períodos históricos ampliamente distinguidos en los estudios de la sociedad chilena del siglo XX por el importante cambio que significaron en el modelo de desarrollo como país y en la vida cotidiana de la población.

El primero de dichos períodos, a partir de la tercera década del siglo pasado, es conocido por el papel de un estado benefactor y desarrollista marcado por la asunción de las capas medias al poder político central; el segundo período, iniciado el año 1973 con la dictadura militar, significó la imposición del libre mercado y la integración a las economías globalizadas como eje del desarrollo del país.

La descripción de las principales transformaciones de la familia que aquí se presenta, ${ }^{1}$ está orientada a analizar los aspectos sociales y culturales más importantes que para el siglo XX destaca la literatura especializada, y que en nuestra opinión, pueden condicionar el proceso de envejecimiento de dos generaciones, entendiendo que a cada una de ellas lo que las distingue es compartir «un mismo cuadro de vida histórico social» (Mannheim, 1990:52). ${ }^{2}$ Esta aproximación se ha rea-

1 Atendiendo a la diversidad social y cultural, en particular por la fuerte desigualdad social que caracteriza a la sociedad chilena, el desarrollo de este tema se realiza procurando evitar que se hable de la familia como referencia a un único tipo de familia. No obstante, en términos sociales concretos, no podemos desconocer que una vez instituida la familia como categoría social y jurídica, la dinámica social tiende a homogeneizar y enmascarar su variedad, de manera que comúnmente el uso de esta categoría contiene expresiones con una fuerte carga ideológica, como lo destaca la investigadora Irma Arriagada (2002). Así, el tratamiento social de la familia termina inscribiéndose en lo que Pierre Bourdieu denomina violencia simbólica. Para este teórico, como en lo fundamental lo postularon varios clásicos pensadores de la realidad social, la familia es el lugar de la acumulación y transmisión del capital económico, simbólico y social, en tanto existiría una estrecha relación entre la familia nuclear tradicional, el estado moderno y las elites dominantes que éste genera. Como sostiene Silvia Anguiano, «El 'tener' una 'familia' es parte inclusive de los requisitos para acceder a determinados mecanismos de transferencias de ingresos monetarios como subsidios de diversas clases (especialmente por parte del Estado)» (Anguiano, 2007:1).

2 La situación definida por el año del nacimiento, en palabras de Mannheim, otorga «la posibilidad que se sigue de participar en los mismos 
lizado centrando el interés en dos ámbitos de la vida familiar de dos generaciones, a saber, las relaciones de pareja y entre padres e hijos.

\section{UNA RESEÑA DE LOS INICIOS DEL PROCESO DE MODERNIZACIÓN EN EL SIGLO XX}

El período anterior a los años treinta del siglo XX, es comúnmente denominado como el período de la exclusión social y del Estado oligárquico, en referencia a los amplios poderes concentrados en un pequeño círculo de la población.

Durante las primeras tres décadas del siglo XX, se agudizaron las demandas sociales de vastos sectores de la población como el inicio de un proceso de modernización en el que el crecimiento de las ciudades potenció el desarrollo de una serie de movilizaciones sociales que culminaron con el desmembramiento del orden oligárquico tradicional. El país fue escenario de la acción de un amplio movimiento social de carácter multiclasista en el que se entremezclaron grandes marchas cívicas, las «marchas contra el hambre», así como propuestas de nuevas políticas y reformas de carácter económico y social junto a directrices para una nueva constitución política. ${ }^{3}$

En este marco social, la diversidad de formas familiares que se arraiga desde los inicios del Estado-nación chileno ha sido tratada por algunos autores como «el desorden de la familia». ${ }^{4}$

acontecimientos, en la misma vida, etc. y, más aún, de hacerlo a partir de una misma forma de estratificación de la consciencia... Sólo un mismo cuadro de vida histórico-social permite que la situación definida por el nacimiento en el tiempo cronológico se convierta en una situación sociológicamente pertinente» (Mannheim, 1990:52-53). Sobre el concepto generaciones, también ver Martín Criado (1998); Feixa (1996); Ghiardo (2004); Gutiérrez y Ríos (2006), entre otros textos.

3 La preocupación por los temas sociales incluyó, no sólo al creciente movimiento popular que exigía reivindicaciones y las demandas de las emergentes capas medias, sino que también a la generación más joven de la oligarquía (Salazar y Pinto, 2002).

4 Con ello se hace referencia a lo común que era en la población encontrar prácticas alejadas de las normas que en materia matrimonial provienen de la colonización española y, consecuentemente, de las normativas de los códigos civiles del siglo diecinueve. «Durante el siglo XVIII, aún en el siglo XIX, la ilegitimidad de un alto porcentaje de recién nacidos, el alto número de parejas unidas consensualmente, la bigamia y el adulterio fue- 


\section{ESCENARIO DE LA GENERACIÓN DE LOS AÑOS 1930-1973: DEL DESORDEN FAMILIAR A LA INSTITUCIONALIZACIÓN DE LA FAMILIA}

\section{a) Politicas sociales, modernización y grupos familiares ${ }^{5}$}

La asunción de las capas medias al poder político significó, en lo fundamental, instalar lo social como una preocupación importante del aparato público. ${ }^{6}$ Entre los principales procesos sociales de este período, cabe señalar una progresiva urbanización, resultado de la migración rural y el proceso de industrialización como base del crecimiento económico. Las políticas de carácter nacional se orientaron a fortalecer el Estado como un agente económico activo en la generación de riqueza así como impulsor de las obras públicas y responsable de un conjunto importante de industrias básicas para el país. Así, el Estado fue constituyéndose en un agente clave para la inclusión social de los crecientes sectores medios y, luego, de los sectores obreros organizados.

En este proceso, con un fuerte énfasis en la promoción de la educación y salud públicas gratuitas, un importante impulso de la inversión en la vivienda social, y legislaciones con mayores regularizaciones en los contratos laborales y el sistema previsional, se desarrolló una serie de políticas sociales que, normalmente respondiendo a un punto de vista masculino, construyeron las condiciones sociales y legales que posibilitaron una vinculación creciente entre la constitución de la familia y el matrimonio, y, en especial, con la familia nuclear patriarcal.

Es interesante observar como en este período de la sociedad chilena se fueron instalando una serie de cambios modernizadores como el acelerado proceso de urbanización, un mayor alfabetismo y la escolarización de la población, así como cambios de orden demográfico. Así, por ejemplo, destaca que hasta el año 1930 la población urbana

ron, entre otras transgresiones a la fe y a la ley, situaciones cotidianas y del común de la gente» (Cavieres, y Poblete, en Valdés, 2004:5).

5 Una consideración central sobre estas realidades, como lo afirma José Olavarría, en sociedades con profundas desigualdades como la nuestra, los cambios que marcan la modernidad y globalización no se inscriben en un proceso único ni homogéneo; menos aún, en el nivel íntimo de las relaciones humanas (Olavarría, publicación virtual: 2).

6 Algunos antecedentes económicos son de interés al respecto: «el porcentaje del gasto social aumentó del $10 \%$ en 1935, al 26,9\% en 1945 y al 28,5\% en 1955» (Valdés, publicación virtual 2004:9). 
en la sociedad chilena no superaba a la rural. Sin embargo, ya en 1952 la población que vivía en ciudades superaba el $60 \%$ y en 1970 ya alcanzaría el $75 \%$. Otro aspecto de interés lo constituye el crecimiento progresivo del alfabetismo y la escolarización. El mayor alfabetismo masculino que hasta la década de 1940 superaba significativamente al de las mujeres, a partir de los años siguientes se anulará dicha diferencia. En términos de los avances de este proceso, en 1907 el alfabetismo alcanzaba a un 40\%, se eleva a un 50\% en 1920; al 75\% en 1952 y alcanza un $90 \%$ en 1970. En orden a los años de escolarización, en 1930 este ascendía a 3,3 años de escolarización promedio de la población mayor de 15 años, alcanzando a 4,5 años en 1960, 6,2 años de escolarización en 1982 y 9,7 años en $1990 .^{7}$

En esta reseña de la modernización del país destacan algunos cambios demográficos. En relación a la tasa de mortalidad, esta alcanzaba a 32 por cada mil habitantes en 1907, desciende a 25 en 1930 y a 10 por cada mil habitantes en 1970 (Brünner, 2006). En este notable descenso, cabe destacar la incidencia decisiva que tuvieron las mejoras en la salud materno-infantil en la primera mitad del siglo XX por la fuerte disminución de la tasa de mortalidad infantil. El aumento de la esperanza de vida al nacer, por otra parte, se incrementa de manera tal que de alcanzar a 30 años en las dos primeras décadas del siglo XX, se eleva a 55 años entre 1920 y 1950, y a 65 años entre 1950 y 1975 (op.cit.).

El proceso social de este período se caracterizó por una serie de políticas sociales dirigidas a instituir la constitución de la familia a través del matrimonio, en lo que se conoce como la «institucionalización de la familia». ${ }^{8}$ Consecuentemente, se sentaron las bases para extender en todos los sectores sociales la familia nuclear patriarcal bajo la concepción moderno-industrial de la familia. Esta tendencia tendrá nuevas evidencias a partir de la década de los años sesenta. En efecto, el discurso del modelo desarrollista otorgó especial importancia al fortalecimiento de la familia, en concreto de la familia nuclear, considerando a esta como un modelo único en las políticas públicas. ${ }^{9}$

7 Porcentajes calculados a partir de datos de INE, Censos 1907-2002 y Ministerio de Hacienda 2005, (Brünner, 2006).

8 Importantes investigadores, Ximena Valdés y Teresa Valdés entre otros, denominan como institucionalización de la familia a la vinculación entre matrimonio y el hacer familia.

9 En este sentido, los trabajos de Elizabeth Jelin (1994) y de Irma Arriagada (2001) destacan la existencia de una debilidad, la que se prolonga hasta el 


\section{b) El orden de las relaciones de pareja}

En este período de la sociedad chilena, en la mayoría de la población la vida cotidiana de familia se caracterizó por transcurrir bajo marcos jerárquicos patriarcales tanto respecto a la vida de pareja como en la relación entre padres e hijos.

Los preceptos sociales prevalecientes estaban dirigidos por «el ideal del salario familiar», esto es, constituir al hombre trabajador como el jefe de familia y el sujeto proveedor y, a su vez, a la mujer como la figura responsable del hogar y la crianza de los hijos. Así, el lugar de los hombres y las mujeres en la familia y en la sociedad, fue favorecido y reafirmado socialmente a través de dispositivos públicos que apoyaban el modelo normativo basado en el matrimonio. ${ }^{10}$ Las definiciones sociales que predominaban en las relaciones entre los géneros, la vida de pareja y la vida cotidiana de familia, se caracterizan por estar inscritas en una estructura jerárquica en la que la vida del hombre está orientada al nivel de lo público y la de la mujer hacia lo privado, la maternidad y lo doméstico. ${ }^{11}$ No obstante, no debe omitirse

día de hoy, en el desarrollo de enfoques actualizados para un diag-nóstico de la heterogeneidad de situaciones que presentan las familias. En el período que se describe, el modelo desarrollista promovía claramente la nuclearización de la familia como correlato del proceso de modernización al que se suponía que se encaminaba la sociedad. Por su parte, como lo consigna Eugenio Tironi (2006), el destacado teórico Talcott Parsons afirmaría que la modernización requiere la disolución de los lazos familiares extensos y la reducción del grupo doméstico a una forma nuclear con uno solo de sus miembros (el hombre) activo económicamente.

10 Es ilustrativo al respecto el siguiente párrafo: «La idea de maternidad moral y del salario familiar se establece en plenitud en Chile con los gobiernos del Frente Popular, culminando el año 1953 con las asignaciones familiares universales a los trabajadores del campo y la ciudad. Pese a que se ha calculado que un tercio de niños y mujeres quedaron fuera del sistema porque sus padres o compañeros no contaban con empleos formales [...] este suplemento salarial llegó a significar cerca del $20 \%$ de los ingresos percibidos por los trabajadores de los años 60 ». (Valdés, publicación virtual 2004:9-10).

11 En relación al nivel cultural, en este último sentido, es interesante el tratamiento hecho por Sonia Montecino en su tesis sobre la importancia del modelo mariano en la cultura chilena. Sin embargo, como sucede con otros temas de la cultura, es indudable que la diversidad y complejidad de las tramas sociales implicadas en las relaciones entre los géne- 
el hecho de que desde los inicios del siglo XX, como una clara señal del cambio que se avecinaba en la sociedad chilena, la movilización de la mujer por sus derechos ciudadanos habría de significar la obtención del derecho a voto en 1949.

Si bien en el orden educacional y civil la mujer tuvo un acceso y ampliación de derechos muy significativo a lo largo de todo el siglo $\mathrm{XX}$, hasta las últimas décadas de dicho siglo no se afectó de manera importante el modelo cultural femenino sobre el que se sustentaban las asimétricas relaciones de género, en las que los permisos para la infidelidad masculina son buen ejemplo de ellas. ${ }^{12}$

Por largas décadas, para la mujer el ejercicio de una sexualidad unida con la maternidad se tradujo en una alta tasa de fecundidad. Será recién en los inicios de la década del 60 , cuando cada mujer tenía en promedio 5,3 hijos, el momento en que comienza un descenso de la fecundidad en Chile (CELADE, 1988). ${ }^{13}$ Este descenso de la natalidad global se presentará de preferencia en las mujeres de ciudades y aquellas de niveles socioeconómicos y educativos medio y alto, y, en pocas décadas, afectará el tamaño medio de las familias.

De acuerdo con José Olavarría y Rodrigo Parrini, ${ }^{14}$ el modelo hegemónico de masculinidad en nuestra sociedad, particularmente fuerte hasta las últimas décadas del siglo XX, ha presentado los siguientes rasgos: ser potente, no expresar emociones, proteger a las mujeres y respetar, en especial, a la propia madre. ${ }^{15}$ Asimismo, establece que el hombre, en la edad adulta, deberá ejercer el control, ser jefe y ejercer la paternidad y por lo tanto ser proveedores aportando el dinero para darle sustento, protección y educación a la familia.

ros constituye, sobre todo desde perspectivas de trabajo de carácter histórico, un campo de trabajo investigativo abierto.

12 Las orientaciones culturales prevalecientes establecían, en este sentido, preceptos en los que la mujer representaba la virtud y, por consiguiente, se entendía que la fidelidad femenina era parte importante del honor familiar.

13 Es de interés consignar que a partir del año 1962, a través del Servicio Nacional de Salud en su programa materno-infantil, se comenzó a aplicar una política de salud destinada a difundir las prácticas anticonceptivas como medio de combatir la alta incidencia de abortos, abarcando cada vez sectores más amplios de la población femenina.

14 Para ambos autores, ver publicación virtual.

15 El uso del concepto de masculinidad/feminidad, aquí refiere a aquellos preceptos asociados con la conducta típica o bien con el modelo hegemónico de los hombres/mujeres en una sociedad o cultura concreta. 
Los cambios que suceden en la segunda mitad del siglo XX tendrán como uno de los procesos más importantes la emancipación de la mujer; por consiguiente, la redefinición de su lugar tradicional en la sociedad será parte de un proceso social y cultural clave que continuará en las próximas décadas.

Sin embargo, las señales de este cambio no siempre serán linealmente consistentes. Es así como a comienzos de los años cincuenta, un importante porcentaje de mujeres trabajaba fuera del hogar, superando las cifras existentes al respecto de inicios de los años setenta (Valdés y Gomariz, 1992). Sin embargo, en una sociedad con profundas desigualdades sociales como la chilena, la siguiente referencia al papel de las mujeres en el movimiento de pobladores de las décadas del cincuenta y del sesenta, ilustra bien que los cambios en los roles tradicionales de género que marcan la modernidad no se inscriben en un proceso único ni homogéneo:

Al examinar las historias de vida de las pobladoras de la década del 60 se constata que ellas fueron más activas, liberadas y experimentadas que lo que sugiere el prototipo «dueña de casa» que los discursos eclesiástico, patriarcal y populista habían diseñado para ellas. Pues, ni fueron madres con dedicación exclusiva a sus hijos, ni esposas que soportaron a sus esposos toda una vida, ni puros modelos supremos de puro estoicismo (Salazar y Pinto, 2002a:253).

Hasta los años setenta, prevalecía de manera importante en nuestro país considerar a la política como una actividad masculina. Así, la participación femenina en partidos políticos en 1970 no superaba el quince por ciento. (Vidal, 1972). En este último sentido, es preciso consignar que a inicios de dicha década, el sentido social para una parte importante de la población masculina estuvo dado, además del hacer familia, por el vínculo a una serie de redes sociales a través de los partidos políticos, los sindicatos, las organizaciones civiles, entre otras. ${ }^{16}$

En la década del setenta, en círculos progresistas y, en especial, en la población más joven, se evidenciaron señales de una importante resistencia a la rigidez de los preceptos sociales de la época. Al res-

16 Esto, en cierta medida, pudo responder a la fuerte gravitación que en esos años adquirió la política en la vida nacional, correlacionada al decisivo papel que en esos años le correspondía al Estado, y a la polarización de las posiciones políticas de la época. En relación a la participación en los sindicatos, ver Salazar y Pinto (2002b:103-104). 
pecto, siguiendo de cerca a Carles Feixa, este proceso adquirió escala internacional, en el que la sociedad chilena no fue la excepción. ${ }^{17}$ En efecto, en la población joven circulaba con fuerza una serie de cuestionamientos que, aunque respondían a dinámicas sociales diferentes, convergieron en contra de los modelos patriarcales y proclamaron una mayor liberación acerca de la sexualidad y la igualdad de los géneros, entre otras realidades. Tras sus iniciales manifestaciones como una suerte de contracultura, dicho proceso derivaría en los años setenta y las décadas venideras en la emergencia de subculturas que defenderían el derecho a la diferencia y a la identidad de varias minorías. Más tarde, dichas minorías habrían de contribuir a dar forma a nuevos movimientos sociales (la liberación homosexual, el ecologismo, el feminismo, el antimilitarismo, etc.) (Feixa, 2005).

\section{c) El orden de las relaciones entre padres e hijos/as: periodo de años 1930-1973}

A lo largo del siglo XX, así como en estos primeros años del siglo veintiuno, la intermediación del Estado ha ido progresivamente transformando lo privado en un tema público. ${ }^{18}$

17 La protesta estudiantil de mayo del 68, en París, constituyó un hito en tanto manifestación inicial de dicho proceso. Tratada como una rebelión generacional de la juventud de esos años, este hecho social reflejaría una búsqueda de alternativas a las instituciones clave de la sociedad de la época (la familia tradicional, la democracia formal, la cultura autoritaria, el positivismo, los medios de comunicación de masas, la monogamia, el productivismo y el armamentismo) en pro, principalmente del pacifismo, la revolución sexual, el valor del subjetivismo y la vida comunal y pastoril.

18 De acuerdo a dos destacados historiadores de la niñez, Philippe Ariès y Lloyd de Mause, aunque con perspectivas teóricas diferentes, las concepciones de la infancia y de los hijos varían según las distintas condiciones sociohistóricas. En su historia de la evolución de las diversas actitudes de la familia hacia los niños, «Ariès se propuso explicar históricamente el tránsito del olvido a la centralidad de la infancia, un tránsito del anonimato y de la indiferencia hacia el niño de las épocas remotas al de una criatura rica en promesas y en futuro, que tenemos hoy en día. Según Ariès, en occidente se ha pasado de una sociedad amplificada en la que el niño, cuando apenas era capaz de valerse por sí mismo, vivía ya como adulto en medio de los adultos, 'libre', en cuanto ser autónomo 
Desde una dimensión social y cultural, ya en los inicios de este período, la modernidad mostraría cambios en un aspecto particularmente importante para la vida cotidiana familiar. Los límites que separaron la vida privada y la esfera pública en la tradicional sociedad chilena, comenzarán a presentar señales de un giro cualitativo que se profundizará a lo largo del siglo XX.

En efecto, de acuerdo con Ximena Valdés (2004), las mediaciones sociales en la vida cotidiana familiar, convertirán al ámbito familiar en un asunto sujeto de crecientes regulaciones jurídico-políticas. En este sentido, en el marco de la profunda desigualdad social en la sociedad chilena, es ilustrativa la siguiente cita respecto a las diferencias en relación con la obligatoriedad de la ley de enseñanza:

Un estudio monográfico realizado a comienzos de siglo XX en una familia popular de Santiago reveló que el aporte de la mujer y los hijos al presupuesto del hogar fluctuaba en torno al 33\%, siendo el más importante el del hijo mayor [...] Por eso, muchos jefes de familia protestaron por el «abuso» de que se dictaran leyes que obligaban a los niños a ir al colegio, cuando lo que todos necesitaban era que trabajaran (Salazar y Pinto, 2002b:63).

La paternidad patriarcal en Chile, respaldada por la legislación hasta 1998 sobre la legitimidad/ilegitimidad de los hijos — establecía que los hijos verdaderamente hijos eran los que nacían en una unión legalmente constituida-, era un modelo de masculinidad hegemónica que, como lo consigna José Olavaria, presentaba la paradoja de permitir a los hombres tener hijos y no, necesariamente, ejercer ni sentirse padres. No obstante, durante este período, por efecto de las políticas sociales que se implementaron, tendrá lugar una tendencia al aumento progresivo de la nupcialidad y, paralelamente, se observará una ostensible disminución de la ilegitimidad en los nacimientos.

El trato diferenciado en la relación entre los padres y sus hijos, según fuere el sexo de los mismos, constituye una constante en la cultura de la época. Así, el grado de separación de lo público y lo privado adquiría formas diferentes con hijos varones que con hijas mujeres respecto de los permisos y las prohibiciones, así como en la división

y productivo, a una sociedad que se encierra en núcleos familiares, privatizando a la infancia y segregándola mediante diversos sistemas 'educativos' que implican la intervención de la autoridad paterna y la vida regulada por regímenes disciplinarios, ya sea en el seno de la familia o en la escuela» (Alzate, 2002:7). 
sexual del trabajo doméstico y no doméstico, y, además, la preferencia por el hijo varón en el caso de optar para la prosecución de estudios de uno de sus hijos. En este marco, en los sectores populares, según lo han constatado diversos estudiosos del tema, el trabajo infantil era parte de las estrategias de subsistencia en la familia popular. En este período de la historia nacional, con bastante frecuencia los niños varones aportaban al debilitado presupuesto familiar, conocidos como los trabajadores infantiles (Salazar, y Pinto, 2002b).

La creciente institucionalización de la vida cotidiana de la infancia a través del colegio, es decir la escolarización de la niñez, se extendió progresivamente hacia los sectores populares. Así, el carácter docente del Estado de la época tenía importantes logros en sus esfuerzos en pro de la obligatoriedad de la ley de instrucción primaria.

Las relaciones entre los padres y sus hijos comúnmente se inscribían en una estructura jerárquica y autoritaria, en especial en relación al padre. Durante este período, prevalecen los preceptos para que la mujer se relacionara con el trabajo reproductivo y el cuidado de los demás miembros de la familia. Las labores de crianza involucraban, por lo común, cercanía y afecto de la madre con hijos/as en sus primeros años. Los varones, por su parte, se mantenían ajenos de participar en el cuidado de los niños así como del trabajo doméstico.

El vínculo entre padres e hijos jóvenes, entre las décadas del treinta al sesenta, se inscribe bajo una clara hegemonía adulta (Salazar y Pinto, 2002b). Sin embargo, en los años sesenta e inicios de los setenta, la relación padres/hijos jóvenes inicia un importante proceso de cambio. El movimiento estudiantil y la juventud tendrán una creciente presencia en la vida nacional.

\section{ESCENARIO DE LA GENERACIÓN DE LOS AÑOS 1970-2007: DE LA INSTITUCIONALIZACIÓN DE LA FAMILIA A SU DES-INSTITUCIONALIZACIÓN ${ }^{19}$}

\section{a) Políticas sociales, modernización y grupos familiares}

La dictadura militar de los años 1973-1990 tuvo un conjunto de efectos que sobrepasó el mundo de lo público. Ella significó, también,

19 De acuerdo con Ximena Valdés (2007), según lo indican las tasas de nupcialidad, el proceso de des-institucionalización de la familia constituye una realidad que en Chile se evidencia a partir de la década de 1980. 
importantes consecuencias en el plano de lo privado y lo íntimo (Valdés y Valdés, 2005).

El libremercadismo neoliberal pasaría a constituir la base del modelo de desarrollo del país que impuso la dictadura militar. La sociedad chilena se habría de encaminar en pocos años, con los efectos sociales que ello implicaría, a formar parte de las economías globalizadas.

Profundos cambios consolidaron el carácter subsidiario del Estado para privilegiar la actividad del sector privado, incluyendo un importante proceso de privatización de las empresas públicas. En esta dirección, como es sabido, disminuyó el importante papel que en tiempo pasado tuvo el Estado y se fortaleció el papel del mercado. Si bien se llevará a cabo una descentralización administrativa del Estado, esta se realiza bajo la lógica del mercado, en ningún caso, de la sociedad civil. Más aún, la siguiente cita lo reseña así: «Mirado en su integralidad, el diseño institucional establecido entre 1974 y 1979, no era sino un proceso de centralización política y administrativa con desconcentración de funciones» (Cumplido, 1983; Vergara, 1991; PNUD, 1993, en Hernández, 1995:189).

La política pública desarrollada en estos años mermó los dispositivos públicos que se convertían en beneficios sociales, característica central del pasado Estado benefactor. Se modificaron la legislación laboral y el sistema de previsión social, y la gratuidad de los servicios públicos de salud, educación y vivienda quedaría focalizada para la población en extrema pobreza. Este conjunto de cambios, como es ampliamente reconocido por la literatura especializada, tuvo efectos importantes en la vida cotidiana de las familias, en tanto se afectaron las bases que favorecieron el tipo de familia nuclear patriarcal.

Así, en este período, si bien se masificó el acceso a mayores bienes y servicios sociales, se ampliaron las desigualdades sociales. Por una parte, la oferta de servicios básicos como educación, salud y seguridad social diversificó notablemente su calidad; por otra, la dinámica excluyente de un mercado de trabajo caracterizado por una heterogeneidad estructural, terminaron por acentuar las diferencias sociales. En consideración de esta realidad, los diagnósticos de organismos internacionales destacaron hace pocos años la conveniencia del desarrollo de programas de protección social: «A pesar de la importancia de promover políticas que generen más y mejores empleos, no cabe esperar que a corto o mediano plazo el empleo sea suficiente como mecanismo de protección de la mayoría de la población frente a riesgos rela- 
cionados con la falta de ingresos, la salud y el envejecimiento» (CEPAL, 2006:3).

En el país, al igual que en la mayoría de los otros países latinoamericanos, esta política económica, como lo destacan especialistas en el tema, ha significado para una gran mayoría el crecimiento de las expectativas de consumo pero con serias dificultades para satisfacerlas. Así, en muchos casos esto significa que para cubrir las necesidades básicas de los grupos familiares, en los sectores populares se han sumado mujeres, jóvenes y niños como aportantes económicos en el hogar. Más aún, como sostiene Irma Arriagada, el contexto social facilita que «se genere una creciente frustración y se promueva la búsqueda de alternativas no lícitas que se expresan en una creciente delincuencia, tráfico de drogas y corrupción entre otros fenómenos de violencia y exclusión social» (Arriagada, publicación virtual:2).

En este marco, la revisión de otras variables en este período muestra que el proceso de modernización iniciado décadas atrás seguirá avanzando en distintos niveles de la sociedad. En efecto, el predominio de la población rural que perduró hasta la cuarta década del siglo XX, dará paso a una urbanización que en el año 1970 se traduciría en el 75\% de la población viviendo en ciudades y en el año 2002 alcanzaría más de un $85 \% .{ }^{20}$ Asimismo, se acentúa el alfabetismo de la población en los años setenta alcanzando un $90 \%$ y en el año 2002 se observa un 96\% de alfabetismo; y, por su parte, los años de escolarización de la población mayor de 15 años alcanzan 6,2 años en 1982 y 9,7 años en 1990 (Ministerio de Hacienda, 2005).

Esta reseña de la modernización de la sociedad chilena no puede omitir el orden de los cambios demográficos. En estos destaca el envejecimiento progresivo de la población. Así es como el aumento de la esperanza de vida al nacer se incrementa de manera tal que sobrepasa los 75 años entre el 2000 y 2005. Sin embargo, como sucede en la mayoría de los países, el envejecimiento de la población resulta, paradojalmente, de la progresiva disminución del peso relativo del segmento menor de 15 años de edad (Pérez Díaz, 2000). En efecto, mientras en la sociedad chilena entre los años 1920 y 1970 el segmento de menores de 15 años no dejó de representar un 40\%, ya en 1982 descendió a $32 \%$ y en el año 2002 no superaba un $26 \%$ (INE, 2000). Por otra parte, no sólo se hace más pronunciado el descenso de la natalidad global iniciado en los años sesenta, se reducen también las dife-

20 Estos porcentajes se han calculado a partir de datos oficiales censales. 
rencias que antes presentaba este indicador entre distintos sectores de la población femenina, en especial según la residencia urbana o rural y los niveles socioeconómicos y educativos (Palma, 2000).

En el orden social, ciertos procesos marcarán decisivamente la vida familiar en las próximas décadas. Entre estos procesos, la incorporación de la mujer al mercado laboral y el envejecimiento de la población repercutirán de manera significativa en los preceptos tradicionales que marcan las relaciones de género y en los arreglos internos de los grupos familiares. Además, la profundización del debilitamiento de la separación entre la vida privada y la vida pública, tendrá como una de sus expresiones que la vida cotidiana familiar sea objeto de mayores regulaciones jurídico-políticas. En este marco, no es extraño que las relaciones de pareja y entre los padres y sus hijos este mediada progresivamente por disposiciones sociales que procurarán, desde la perspectiva de los derechos ciudadanos, principalmente proteger a la mujer y los niños.

En estos últimos años, la política pública ha desarrollado una reorientación poniendo especial énfasis en promover una mayor igualdad de oportunidades por la vía de la educación, por la vía del gasto social y la promoción del pleno ejercicio de la ciudadanía. ${ }^{21}$

Sobre la grave desigualdad social del país, otros estudiosos postulan, sin embargo, que si bien este proceso estaría marcado por la integración del país a las economías globalizadas a través del modelo neoliberal, es plausible que se trate de un complejo proceso social que está afectando no sólo la calidad de la convivencia familiar sino también el sentido comunitario de los individuos. Una alerta a la sustentabilidad social de la modernización hecha por Norbert Lechner (1999), otorgaba atención especial a la importancia de lograr un desarrollo de la subjetividad que sea complementario ante el avance modernizador. Por su parte, Arriagada y Godoy (1999), en relación a las economías latinoamericanas y la globalización y su impacto en la vida familiar y

21 Una importante manifestación social asociada a esta reorientación en la gestión pública estuvo dada por la movilización estudiantil de 2006 encabezada por estudiantes secundarios de Chile, conocida como la revolución de los pingüinos. Se estima que esta movilización habría contado con una adhesión de más de 600.000 escolares, constituyendo la mayor protesta de estudiantes en la historia de Chile. Las peticiones reclamadas por los estudiantes se relacionaron con la derogación de la Ley Orgánica Constitucional de Enseñanza, el término de la municipalización de la enseñanza y la solicitud de un estudio para la reformulación de la jornada escolar completa. 
comunitaria, ponen énfasis en la importancia de las condiciones de alta inseguridad, precariedad y exclusión social de parte de la población. Para otros teóricos, las comunidades ya no están unidas sólo por la tradición, sino por una paradójica colectividad en la que se instaló un individualismo institucionalizado (Beck-Gernsheim y Beck, 2003).

\section{b) El orden de las relaciones de pareja}

La política económica que supone el modelo de desarrollo de las últimas décadas creó, como ya se reseñara, condiciones que erosionaron el «ideal del salario familiar», base de la prevalencia de la familia nuclear patriarcal. Por una parte, con frecuencia los ingresos del hombre trabajador y proveedor de recursos serán insuficientes para cubrir el sustento y las crecientes necesidades de los miembros de la familia. Por otra, se han generado progresivamente condiciones que promueven que la mujer trabaje fuera del hogar.

Las bases sustentadoras del modelo tradicional de familia se vieron debilitadas con los cambios en el orden económico del país. Esto ha derivado, en especial, en un cuestionamiento del precepto tradicional respecto del lugar de los hombres y las mujeres en la familia y en la sociedad. Los imperativos de orden económico, sumados los avances del movimiento social de las mujeres en relación a la igualdad de oportunidades (mayores niveles de educación y derechos cívicos, separación de sexualidad y maternidad, entre otros), han impulsado prácticas para contar con un segundo ingreso en el hogar. ${ }^{22}$ Asimismo, quedó en evidencia que se transita hacia un cambio del marco jerárquico de autoridad masculina.

En estos años, la separación de la sexualidad y la maternidad se profundiza notablemente en los distintos sectores sociales. La mujer ha privilegiado los estudios y el trabajo, presentando una tendencia creciente a una maternidad tardía. ${ }^{23}$

22 De acuerdo al INE, durante el trimestre diciembre 2005 a febrero 2006, la tasa de inserción femenina en el mercado del trabajo alcanzó un 38\%, el mayor nivel que ha existido en la historia.

23 En relación al tema de la fecundidad y política pública, cabe mencionar que en fecha reciente, como parte de la reforma previsional del 2007, se ha propuesto otorgar un bono por cada hijo nacido vivo consistente en $10 \%$ de dieciocho salarios mínimos, el que se agregará al fondo de pensión de vejez de la mujer. 
Si bien en estas últimas décadas la sociedad chilena muestra un progresivo aumento de la participación de la mujer en el trabajo fuera del hogar, aun cuando presenta tasas de participación en el mercado laboral inferiores al promedio de los países latinoamericanos. ${ }^{24}$ Actualmente, en comparación con los hombres, las mujeres todavía se concentran en empleos de menor calificación y remuneración.

En un marco cultural en el que el modelo hegemónico de masculinidad implica ser jefe de hogar en tanto se es proveedor, la incorporación de la mujer como un nuevo aportante de ingresos al hogar constituye un factor que erosiona las bases sobre las que se sustenta la autoridad del hombre en el hogar. ${ }^{25}$ Los patrones de funcionamiento de los hogares transitan hacia redefiniciones de roles en la pareja, y esto, comúnmente, no está exento de conflictos en el interior de las familias.

En este período de la vida nacional, inicialmente derivado por las prohibiciones impuestas de la dictadura militar, es común que la población masculina haya restringido su participación en otras organizaciones que no sean aquellas relacionadas con el trabajo, centrando en la familia parte importante de su sentido social. Como lo plantea José Olavarría, ${ }^{26}$ esto adquiere especial vigencia en los sectores populares, donde paradojalmente la condición de proveedor del hombre, elemento clave de su identidad, es más vulnerable al contar con trabajos más precarios y con menos recursos. En sectores medios y altos, factores tales como mayores niveles de escolaridad y contar con una más amplia diversidad de capitales, le permitirían al hombre insertarse en otras redes sociales de modo que la identidad masculina no se estaría jugando con la misma intensidad en la autoridad con su pareja ni en el ejercicio del ser jefes de hogar.

Otra de las expresiones más significativas de las últimas décadas en torno a la familia es la ampliación de formas para las uniones de pareja. Conocido como la des-institucionalización de la familia, este

24 En Chile, según datos del INE, en 1976 trabajaba un 25\% de las mujeres que tenían 15 y más años; en 1990 esa proporción ascendía al 31,8\% y, hoy en día, se ha elevado a poco menos del $40 \%$. Las tasas de participación en los hombres oscilaban entre un $70 \%$ y poco menos del $80 \%$ en los mismos años.

25 El concepto de masculinidad se trata como una construcción cultural en la que el contexto histórico y socioeconómico condiciona las particularidades de los preceptos sociales que se le asocian, y, por consiguiente, dicho contexto condiciona, también, las crisis y conflictos que ello implica.

Ver al respecto, Olavarría, publicación virtual a. 
proceso consiste en la creciente separación entre la constitución de la familia y las formas jurídicas del matrimonio. Es así como, las uniones consensuales de pareja, más comunes en el pasado en los sectores populares, se extienden en el presente en los estratos de mayor nivel educacional. ${ }^{27}$ Asimismo, hoy en día, los hijos nacidos fuera del matrimonio han superado a aquellos que nacen dentro del mismo, y los hogares con jefatura femenina, por su parte, alcanzan al 21,6\%. Este porcentaje se eleva a $37 \%$ en los hogares indigentes y a $32 \%$ en los hogares pobres (INE, Censo 2006).

Las relaciones entre el hombre y la mujer están susceptibles a un cambio en relación a la autoridad masculina en la pareja y en el hogar. Eugenio Tironi (2006), plantea que la acentuación del proceso de modernización en los últimos treinta años ha dado lugar a tres nuevas tendencias en la familia: la diversificación de las formas de familia, la desinstitucionalización del vínculo matrimonial y la des-diferenciación de la división sexual del trabajo. A continuación, algunas muestras de cambios jurídicos para las relaciones entre el hombre y la mujer. En el año 1989 se modificó el código civil eliminando la obligación legal de obediencia de la mujer al cónyuge; la ley que en el año 1994 sanciona como falta la violencia doméstica, da paso en el 2005 a una nueva ley de violencia intrafamiliar, la que eleva su penalización al otorgarle calidad de delito; y, además, en el 2004, se flexibilizó la ley de matrimonio civil incorporando la posibilidad de divorcio.

En la actualidad, hay una multiplicidad de formas familiares en lo que destaca el incremento de hogares monoparentales y, en estos, más del $80 \%$ con jefatura femenina (Mideplan, Casen 2006). Además, como parte del proceso de envejecimiento del país, han aumentado los hogares unipersonales y, en muchos casos, también, grupos familiares constituidos por cuatro generaciones. Por otra parte, aun cuando se observa un proceso de diversificación de patrones familiares, las familias nucleares aún son predominantes, pese a que en la última década se aprecia una leve disminución de ellas.

Como una realidad asociada con la mayor esperanza de vida, en particular cuando se suma a mejores niveles de ingresos, se ha facilitado un incremento de la paternidad en hombres mayores así como el

27 Este fenómeno se extiende a los otros países latinoamericanos. La consensualidad como una forma de unión de pareja cada vez cuenta con mayor aceptación social, asimismo, esta sería similar en las zonas urbanas y rurales (García y Rojas, 2002). 
incremento de nuevas parejas ha dado lugar a la formación de familias reconstituidas o reensambladas, aquellas que incluyen a hijos de uniones anteriores. Asimismo, en directa relación con el envejecimiento de la población chilena, es de interés señalar que, de acuerdo a datos recientes, la jefatura del hogar de personas mayores alcanza al $29 \%$ de los hogares (op.cit.).

En los últimos años, la vida cotidiana familiar ha incorporado elementos que relativizan los marcos jerárquicos de la familia tradicional y se han instalado señales de inicio de un proceso de democratización de la vida privada pero, a su vez, se está en presencia de una crisis que, en tanto hace más compleja la trama de representaciones simbólicas, tiene una importante repercusión en la vivencia subjetiva, en el sentido de la intimidad de las personas, en las identidades y las relaciones de género. ${ }^{28}$

\section{c) El orden de las relaciones entre padres e hijos/as}

En estas últimas décadas, en el marco de la modernización y la globalización en pleno desarrollo, varios procesos afectan, como veremos a continuación, las relaciones entre padres e hijos.

El impacto de la dictadura militar en la sociedad chilena, como lo destaca la literatura sociológica, no sólo estuvo en la esfera de lo público, también se expresó en el orden de la intimidad. En este último sentido, como sostiene Teresa Valdés (2005), el discurso y la agenda normativa-conservadora que predominó en esos años, entre otra de sus expresiones, se tradujo en la eliminación de la educación sexual de los colegios y en los consultorios de salud.

La intervención del Estado ha profundizado en estos últimos años la transformación de lo privado en un tema jurídico-público. Este proceso que intercede la relación entre padres e hijos, en el año 2007 significó incluir una indicación para dar lugar a un proyecto ley que modifique el Código Civil procurando prohibir los castigos físicos a

28 La tendencia hacia una igualdad de género, igualdad en la diversidad, considerando a hombres, mujeres, homosexuales, bisexuales, lesbianas, travestis, etc., bajo los valores de igualdad, libertad y autonomía, muy probablemente será parte de un proceso de avances y retrocesos en una sociedad en la que, como expresiones de una masculinidad hegemónica homofóbica y patriarcal, prevalece con fuerza la discriminación y la inequidad de género. 
los niños a través de la sanción a los padres. No obstante, la socialización del niño mediante correctivos corporales es una práctica que en nuestra cultura se repite por generaciones.

En el orden económico, el modelo de desarrollo neoliberal ha socavado y terminado con el «salario familiar» como pilar de la familia nuclear patriarcal $\mathrm{y}$, también, ha impulsado la incorporación de la mujer al mercado laboral. Estas realidades, junto al proceso de envejecimiento de la población, constituyen en la actualidad procesos clave para indagar en torno a la variedad de arreglos que ocurren en la familia.

Entre otras realidades que afectan significativamente los vínculos paterno-filiales, destaca la extensión de la escolarización en una suerte de institucionalización de la infancia y de la juventud. La extensión de la escolarización en la juventud, sin embargo, varía fuertemente según el estrato socioeconómico de los jóvenes. Así, según el Instituto Nacional de la Juventud (INJUV), en los sectores de mayores recursos los jóvenes dedicados al estudio, 66\%, duplican a los de sectores más desfavorecidos (INJUV, 2007). Esto significa que la juventud del sector popular, en tanto etapa de preparación institucionalizada, para muchos puede ser una clase de edad inexistente. ${ }^{29}$

El proceso de escolarización de la infancia y la juventud muestra que, a las leyes de obligatoriedad de la instrucción primaria de los años veinte, se suma una serie de modificaciones legales que culminan con la promulgación de la ley sobre enseñanza media obligatoria del 2002, que eleva a doce años la escolaridad para los jóvenes menores de veintiún años. Se agrega a esto, por otra parte, una institucionalización cada vez más temprana de la niñez a través de una serie de otras organizaciones (kinder, pre-kinder, playgroup, salas cuna). Sin em-

29 De acuerdo a Enrique Martín Criado, el concepto clase de edad «nos remite, en un momento del tiempo, a la división que se opera, en el interior de un grupo, entre los sujetos, en función de una edad social: definida por una serie de derechos, privilegios, deberes, formas de actuar... - en suma, por una 'esencia social'- y delimitada por una serie de momentos de transición — que difieren históricamente: matrimonio, servicio militar, primera comunión, certificados de escolaridad-. A su vez, cada grupo social establece una serie de normas de acceso - más o menos codificadas y ritualizadas en forma de 'ritos de paso' - de una clase de edad a otra. Esta división de clases de edad, por tanto, es variable históricamente [...] que se construye en el seno de cada grupo social en función de sus condiciones materiales y sociales de existencia y de sus condiciones y estrategias de reproducción social» (1998:86). 
bargo, en el tránsito a la modernidad y la globalización, se vive la paradoja de que, a raíz de la multiplicidad de información y de agentes socializadores, cada vez es mayor el desfase de la escuela con respecto al mundo de los educandos. En este marco, las nuevas tecnologías de la informática y las comunicaciones constituyen un indiscutido nuevo agente de socialización, cuyo nivel de impacto aún es un tema en investigación para los especialistas.

El tradicional espacio de dominio del padre, se ha desdibujado de modo que ya no es extraño el desarrollo de relaciones menos autoritarias. Al sello de obediencia que en el pasado caracterizaba el vínculo del hombre con la mujer, se ha agregado de manera creciente que la sociedad interceda y avance hacia una mayor protección de la mujer y los hijos. En este sentido, las definiciones que provienen desde la esfera jurídico-pública constituyen un reflejo del proceso de transformación que tiene lugar en el sistema de edades. Muestra de ello es la erosionada práctica de la autoridad paterna como vehículo para resolver los conflictos al interior de la familia.

En el marco de la incipiente tendencia democratizadora de las relaciones entre padres e hijos, inscritas en una estructura cada vez menos jerárquica y autoritaria, la antigua sumisión de los hijos está dando paso a relaciones más igualitarias. Este cambio, sin embargo, como lo destacan algunos autores, José Olavarría entre ellos, el vínculo padres/hijos en muchos casos presenta relaciones que están tensionadas por el ejercicio de autoridad para imponer normas y valores, por una parte, y la expresión de afecto e intimidad, por otra.

En la población más joven con más frecuencia se cuestionan los modelos patriarcales y se proclama una mayor apertura y liberación acerca de los preceptos sociales de género y sexualidad. Sin embargo, estas transformaciones son dispares y heterogéneas. La literatura especializada destaca que las diferencias en el acceso a una mayor variedad de recursos culturales según el nivel socio-económico, constituye un factor importante en las disposiciones personales de la población más joven en los temas de sexualidad y de las identidades y las relaciones de género. De acuerdo con Rodrigo Parrini (1999), en sectores con bajos niveles educativos, en la identidad de la mujer muchas veces prevalece con fuerza su relación con lo materno y en la identidad del hombre, la relación con la paternidad. Pero, esto no significa desconocer que también en los otros sectores sociales nuestra base cultural establece que el peso de la paternidad en torno a la identidad masculina sea decisivo en la reproducción simbólica y la definición de un 
lugar social para los hijos. En este mismo sentido, hay que agregar que, dada la significativa prolongación de la vida de la población, en muchos casos los abuelos y bisabuelos encarnan la memoria familiar y colectiva y, por consiguiente, ellos juegan un importante papel en relación a la identidad social de los más jóvenes.

En relación a la nueva generación, destacan las prácticas que indican una iniciación más temprana en la sexualidad activa y un incremento del embarazo y de la maternidad/paternidad adolescente. Según estudios recientes, casi un tercio de los jóvenes ha vivido un embarazo no planificado. En este sentido, las diferencias socioeconómicas son decisivas: en el segmento de mayores recursos esta realidad representa un $9,8 \%$; en los sectores de menores ingresos los porcentajes superan un $37 \%$ (INJUV, 2007).

El cambio en la legislación sobre legitimidad/ilegitimidad de los hijos, año 1998, constituye otro factor más de debilitamiento del modelo de masculinidad tradicional.

Asimismo, la tradicional importancia de la familia como agente de socialización y enculturación, como la típica unidad social encargada de reproducir el marco valórico y el ordenamiento jerárquico, hoy en día es escenario de crecientes tensiones y contradicciones. Por un lado, los padres, en especial en los sectores populares, en su mayoría con menor nivel de escolaridad que los hijos, se ven expuestos a un debilitamiento de su autoridad. Asimismo, el grado de pobreza del grupo familiar participa en que en este segmento social se considere, también, a los hijos como aportantes de recursos al ingreso familiar. Por otro lado, estudios de fines del siglo XX destacan las expectativas que los padres de sectores populares tienen con sus hijos/as y los mismos confirman que los padres tienen como gran anhelo que estos lleguen a ser más que ellos (Valdés y Olavarría, 1998).

A pesar de observarse un avance hacia un trato más igualitario según el género, en la relación entre padres e hijos aún prevalece un trato diferenciado con hijos varones y con hijas mujeres respecto a las normas y reglas para desenvolverse en el espacio público y privado, los permisos y las prohibiciones, y la división sexual del trabajo doméstico. No obstante, como veremos más adelante, algunos cambios en el comportamiento de los jóvenes parecen ya instalados.

Efectivamente, respecto de esto último, hoy en día destaca la temprana iniciación sexual de los jóvenes; una tendencia general hacia una maternidad tardía y el retraso en la edad en que establecen unio- 
nes de pareja. ${ }^{30}$ Así, en los últimos años, un tema de amplio debate entre las autoridades eclesiásticas y las de gobierno han sido las medidas adoptadas para disminuir el mayor número de embarazos que se concentra en adolescentes de los sectores populares. ${ }^{31}$ Según INJUV (2007), el embarazo no planificado en jóvenes entre 15 y 19 años de edad alcanza a un promedio del $15,9 \%$; y, en el segmento más precario al $36,1 \%$.

A fines del siglo XX se constató una importante variación en la condición de los jóvenes en el hogar, de manera que descendió significativamente el número de jóvenes en situación de jefes de hogar (INJUV, 2000). Esto último, junto a la dificultad de los jóvenes de independizarse de la familia de origen, forma parte, aunque por razones distintas, de un proceso de prolongación de la juventud y de otras etapas del ciclo de vida, tales como la adultez y la vejez.

Asimismo, en estos últimos años cabe destacar el énfasis que en la literatura especializada ha adquirido el hecho de que en muchas familias, en su mayoría de sectores populares, los jóvenes no encuentran trabajo dignamente remunerado. De acuerdo a los datos oficiales, en el 2007 , en un contexto de un $7 \%$ de cesantía como promedio nacional, en los jóvenes entre 15 y 24 años de edad esta condición se elevó al 19\%. No obstante, el problema de la inserción laboral de los jóvenes constituye un tema prioritario en la sociedad chilena así como a nivel latinoamericano y a escala mundial (Charlín y Weller, 2006) En este contexto, las familias con frecuencia deben hacer frente al alcoholismo y la drogadic-

30 De acuerdo al Instituto Nacional de la Juventud, Encuestas Nacionales de Juventud (1994, 1997, 2000, 2003 y 2006), cada vez es menor la edad promedio de inicio de la vida sexual y se comportan más precozmente los jóvenes que viven en condiciones más precarias. Además, hay un bajo uso de métodos anticonceptivos en los jóvenes entre 15 y 18 años de edad.

31 Las nuevas normas de fertilidad permiten la facilitación por el sistema nacional de los servicios de salud pública de la píldora del día después a las jóvenes a partir de los 14 años, sin el consentimiento de sus padres. Sobre esta realidad, según informa la Sociedad Chilena de Obstetricia y Ginecología (publicación virtual), los antecedentes indican que Chile figura en el lugar número 18 de los países con la mayor tasa de fecundidad en adolescentes, según estadísticas de la Organización Mundial de la Salud. Lo que se traduce en que, de los 235 mil nacimientos que ocurren en promedio cada año, un $15 \%$ - 32.250 - son hijos de madres cuya edad está entre 15 y 19 años. 
ción de alguno de sus hijos/as, o bien, como se expuso más arriba, al embarazo de una hija adolescente. Estas situaciones familiares de tensión y conflicto, estarían facilitando que en las relaciones paternofiliales surja la confrontación y la trasgresión de normas (PNUD, 2002).

Finalmente, aunque con un desacuerdo entre los especialistas respecto de la profundidad del fenómeno, se postula que en nuestra sociedad, como un signo de los cambios ocasionados por la modernización y el proceso de globalización, habría un desarrollo incipiente de procesos de individuación que confirman el derecho individual por sobre el familiar, en los cuales se realza la realización personal por sobre los intereses familiares. Según la tesis sostenida en el Segundo Informe del Desarrollo Humano en Chile (PNUD, 1998), la vertiginosidad de los cambios de la sociedad chilena han producido transformaciones que orientan a las personas a organizar sus vidas en forma más individual, imponiendo una ética de autorrealización y del éxito individual. Por consiguiente, la capacidad de los individuos para escoger por sí mismos el tipo de vida que desean habría aumentado dado que los valores tradicionales constituirían opciones y no obligaciones. Sobre este tema es de interés consignar que para algunos especialistas la aceptación de nuevas formas de constitución y funcionamiento de las familias con posibilidades de autonomía y de reflexividad de todos sus miembros en la toma de decisiones, sería expresión de la propia modernidad (Arriagada, 2002). Otros, aunque ponen énfasis en aspectos diferentes, cuestionan la eficacia de la familia como un refugio ante los avatares de la modernidad, dado que el problema central radicaría en que estas no cuentan con los recursos cognitivos, materiales y de sociabilidad para hacer frente a los nuevos problemas a los que están enfrentadas (Güell, 1999). Teresa Valdés, José Olavarría y Ximena Valdés, en varios de sus escritos, por su parte, sitúan su preocupación en las contradicciones que presenta la coexistencia de patrones patriarcales y otros más igualitarios, y en la importancia del factor socioeconómico respecto de las distintas visiones de mundo y estilos de vida por diferencias ante los influjos culturales de la modernidad.

\section{COMENTARIOS FINALES}

A la luz de la revisión y análisis de los profundos cambios políticos y económicos acaecidos en la sociedad chilena y su impacto en la familia, es posible realizar algunas reflexiones en torno a las transformaciones en la subjetividad social de las generaciones del siglo XX o, en 
otros términos, a los cambios en la situación de generación o en el habitus generacional de la edad. ${ }^{32}$

i) El tránsito de una cultura tradicionalmente patriarcal hacia un nuevo contexto social en el que comienzan a mostrarse los efectos de la modernidad y la globalización, aunque aún balbucientes, está acompañado de importantes consecuencias en las distintas dimensiones de la sociedad. Entre estas últimas, destaca la tensión creciente a que está siendo sometido el ordenamiento jerárquico de la vida cotidiana. En particular, se visualizan señales de cambio, entre otros ámbitos de la vida social, en dirección a relaciones más igualitarias en el sistema de género y entre las distintas edades.

ii) Las esferas de dominio que han predominado hasta hoy en día para el hombre y la mujer comienzan a ceder en su rigidez de antaño y pasan a formar parte de un proceso de cambio cuya multiplicidad de efectos no siempre serán predecibles. En este sentido, sin embargo, las nuevas formas de estratificación social y las diferencias según se acceda o no a los beneficios y las oportunidades que se abren con la globalización, muy probablemente habrán de constituir un nuevo escenario social con profundas desigualdades sociales en el que se instale y coexista una gradiente heterogénea de situaciones de dominio/sumisión y de cooperación/autonomía en las relaciones de género.

No obstante, si bien para parte importante de la población la vida cotidiana avanza hacia el entrelazamiento de nuevas formas de cooperación en las relaciones entre el hombre y la mujer, este proceso no está exento de conflictos y contradicciones. Así también, con los roles de paternidad/maternidad.

iii) En nuestra sociedad, la prevalencia de relaciones de género apoyadas en exigencias propias de los roles familiares tradicionales

32 De acuerdo al PNUD (1998), por subjetividad social se entiende al ámbito donde se van constituyendo los sujetos, esto es el mundo de las emociones, percepciones, motivaciones, representaciones, reflexiones y voluntades. Como lo recuerda Ghiardo (2004), para Mannheim (1990), una misma situación de generación es el punto donde se unen el tiempo histórico y las condiciones sociales e históricas de existencia. Una interesante profundización en este sentido, nos la da Patricio Ríos con la conceptualización habitus generacional de edad. Esta última, entre otras precisiones, enfatiza que «las coincidencias de época y de una misma posición en la estructura social, permiten postular prácticas, sensibilidades, posturas, gustos, tendencialmente coincidentes, es decir habitus coincidentes, habitus generacionales» (documento inédito). 
pero sostenidas en un escenario social diferente, constituye una fuente importante de conflictos y tensiones para la población. Así, por ejemplo, la identidad masculina jugada en importante medida en ser proveedor y ejercer la autoridad, bajo las condiciones que impone la economía neoliberal (precariedad del empleo, insuficientes remuneraciones y creciente demanda familiar de bienes y servicios), para muchos casos constituye una base cierta de crisis, en especial, de la masculinidad popular.

Más aún, el impacto que en el modelo hegemónico de masculinidad están representando los cambios económicos y sociales —entre otros, el proceso de incorporación de la mujer al trabajo y al espacio público en general, los nuevos procesos de socialización de las generaciones jóvenes y la instalación y profundización del discurso de los derechos de mujeres y niños-, tiene repercusiones claras. En efecto, además de crear nuevas condiciones al interior de las familias en la cotidianidad de las relaciones de género y entre padres e hijos, dichos cambios están afectando la vigencia del modelo tradicional de masculinidad y favoreciendo la construcción de nuevos modelos de masculinidad y, por consiguiente, profundizando el proceso de cambio del modelo tradicional de feminidad.

iv) El debilitamiento del peso de las convenciones tradicionales en los distintos ámbitos sociales e institucionales, junto al cambio en los procesos formativos de las nuevas generaciones y la profunda transformación social y cultural que representa el cambio en la posición de la mujer en la sociedad, constituyen un contexto social que hace más compleja la trama de relaciones en el sistema de edades. Esto último, tanto en relación a las características que adquiere la construcción social de las edades como respecto a la construcción generacional de la cultura. ${ }^{33}$

Respecto del sistema de edades, es de interés destacar que la dinámica social tiende a enmascarar la variedad y heterogeneidad que hay en la sociedad en relación a las distintas edades. En la medida que éstas, en muchos de sus aspectos constitutivos, son instituidas como categorías sociojurídicas, el tratamiento social de una y otra edad, en uno u otro estrato social, se sostiene de acuerdo a los preceptos dominantes y, consecuentemente, en la vigencia de modelos hegemónicos

33 Para Carles Feixa (1996), la combinación de ambas perspectivas de análisis constituye un paso clave para profundizar los estudios sobre las edades. 
de las edades. Así, esta realidad en nuestra sociedad constituye una importante expresión de violencia simbólica.

v) Una somera revisión respecto de la situación de la vejez nos informa al respecto. En este sentido, las tradicionales estigmatizaciones de que ha sido objeto este grupo etáreo constituyen expresiones de un marco cultural que invisibiliza sus capacidades y derechos. Así, el envejecimiento de la actual generación de mayores, con esperanzas de vida muy superiores a la generación que les antecedió, ocurre en un contexto social que, a pesar de las nuevas condiciones asociadas al envejecimiento de la población, se caracteriza por la exclusión social y la discriminación de la persona mayor. Sin embargo, el modelo tradicional de la vejez es tensionado por los desafíos y las nuevas demandas que acompañan al envejecimiento de la sociedad. En este marco, hay atisbos de la emergencia de una nueva perspectiva en el campo del envejecimiento y la vejez que visualiza la etapa de la adultez mayor a partir de la capacidad y del derecho a formar parte y a ejercer la ciudadanía activa en la comunidad a la cual pertenece. Pero, a la luz del carácter de la mayoría de los programas sociales de distintas instituciones dirigidos a este grupo etáreo, predominan preceptos tradicionales que se traducen en un trabajo que comúnmente trata a este segmento de la población como un recurso inexistente para el desarrollo y la integración social.

vi) La profunda desigualdad social que caracteriza al actual modelo de desarrollo del país y las diferencias en la estructura de oportunidades que se abren con la globalización tiene importantes consecuencias para las distintas clases de edad en los diferentes estratos sociales. Sin embargo, a modo de ejemplo, no es extraño que el trato que la sociedad ofrece a la juventud se apoye en visualizaciones que homogenizan a este segmento de la población. Si bien la escolarización es un proceso que se ha acentuado en las últimas décadas para la juventud, en los sectores más postergados esta es una etapa de la vida que en muchos casos, si no llega a ser como en antaño una clase de edad inexistente, presenta contenidos muy diferentes a la serie de derechos, obligaciones y comportamientos que establece el modelo hegemónico de juventud. ${ }^{34}$

34 En el entendido que se trata de una construcción cultural en la que el contexto histórico y socioeconómico condiciona las particularidades de los preceptos dominantes, en la sociedad chilena, de acuerdo a la literatura especializada, prevalece una visualización de la juventud como la 
vii) La preocupación creciente respecto de prácticas de temprana iniciación en una sexualidad activa, en particular respecto del incremento del embarazo y la maternidad/paternidad adolescente, es una manifestación del desborde de los límites establecidos por las convenciones. En especial, hay un desbordamiento por el cambio de los sentidos subjetivos de la sexualidad e intimidad en los más jóvenes, incluso en los niños. Ello repercute con particular fuerza en las relaciones entre padres e hijos y, en general, entre una y otra generación.

viii) Las nuevas formas de socialización que acompañan a la modernidad y la globalización, junto al mayor nivel de escolaridad alcanzado por los jóvenes hoy en día, han debilitado la autoridad patriarcal y han aumentado la distancia entre una y otra generación. En este sentido, como es sabido, las fronteras familiares son tensionadas, por una parte, por el peso que representan las variadas demandas que implica en la actualidad el desarrollo y la autonomía de la generación joven y, por otra, por una prolongación de la dependencia filial, la que en los últimas décadas preferentemente en los estratos medio y alto se ha extendido significativamente. Así, en muchos casos no resulta extraño que los intereses personales de los jóvenes rompan con las expectativas paternas en relación a su contribución con los intereses familiares. Tampoco resulta raro que, a su vez, en el ejercicio de la paternidad las prácticas de los jefes de hogar muestren la aceptación de una esfera de menor dominio e influencia paterna.

ix) En este marco, las experiencias juveniles de varones y mujeres, aunque muchas veces tengan un carácter experimental, reflejan

etapa de la vida en la que la responsabilidad central del joven es el estudio y la preparación para su futura vida laboral. A lo anterior se agrega, asimismo, una cierta permisividad social para que los jóvenes desarrollen comportamientos experimentales en distintos ámbitos de la vida cotidiana, $y$, en este proceso, se inicien en una vida sexual activa. Además, aún cuando estas particularidades varían según se trate de un joven o una joven, se le considera como una fase en la que, en su desarrollo hacia una independencia y autonomía, no son extraños las crisis y conflictos. Se identifica, también, que una fuente de creciente tensión en la actualidad, dice relación con la prolongación de su dependencia con el hogar paterno. Este tema no es materia para ser profundizada en esta ocasión; no obstante, es preciso consignar a lo menos que, en el país, la política pública dirigida a este segmento de la población, y por lo tanto, el Instituto Nacional de la Juventud, ubica la juventud entre los 15 y 29 años de edad. 
nuevas orientaciones valóricas que en varios ámbitos rebasan los límites de las actuales normas y convenciones. Sin embargo, estas prácticas sociales no están exentas de dificultades para la constitución de sus propias identidades etarias puesto que, históricamente, las nuevas generaciones se formaron al interior de la familia y la comunidad, por contraste con sus mayores. Hoy en día, el escenario social ha cambiado y, lo más importante, el común patrón cultural de una y otra generación se ha resquebrajado. En este sentido, la coexistencia de patrones tradicionales junto a los efectos diferenciados de las influencias culturales de la modernidad (con distintos elementos en las visiones de mundo, estilos de vida, entre otros), además de agregar complejidad al proceso de construcción social de las edades, establece un escenario con nuevas contradicciones para la hegemonía adulta en la construcción generacional de la cultura.

SANTIAGO (CHILE), ENERO 2008

RECIBIDO: MARZO 2008 ACEPTADO: ABRIL 2008

\section{REFERENCIAS BIBLIOGRÁFICAS}

AlZATE, MARÍA Victoria (2002): «El 'descubrimiento' de la infancia: historia de un sentimiento». Revista de Ciencias Humanas №30. Bogotá: www.utp.edu.co.

ANGUIANO, Silvia (2007): «Las estrategias de reproducción familiar».

En: www.revistakairos.org.

ARRIAGADA, IRMA (2005): «Transformaciones sociales y demográficas de las familias latinoamericanas». En XIMENA VALDÉS y TERESA VALDÉS (editoras): Familia y vida privada. Santiago: FLACSO, CEDEM y UNFPA. (2004): «Estructuras familiares, trabajo y bienestar en América Latina». En: Cambio de las familias en el marco de las transformaciones globales: necesidad de políticas públicas eficaces. Reunión de expertos, 28 a 29 octubre de 2004. Santiago: CEPAL.

- (2002): «Cambios y desigualdad en las familias latinoamericanas». Revista de la CEPAL $\mathrm{N}^{\circ} 77$. Santiago: CEPAL.

(2001): «Familias latinoamericanas. Diagnóstico y políticas públicas en los inicios del nuevo siglo». Serie Políticas Sociales No57. Santiago: CEPAL.

——Nuevas familias para un nuevo siglo?» En: www.socialwatch.org. 
— y LORENA GODOY (1999): «Seguridad ciudadana y violencia en América Latina: diagnóstico y políticas en los años noventa». Serie $C E-$ $P A L / E C L A C \mathrm{~N}^{\circ} 32$. Santiago: CEPAL.

BECK-GERNSHEIM, ElisABETH y UlRICH BeCK (2003): La individualización. El individualismo institucionalizado y sus consecuencias sociales y políticas. Barcelona: Paidós.

Bourdieu, PIERRE (1999): «El espíritu de familia». En Razones prácticas. Sobre la teoría de la acción. Barcelona: Anagrama.

BRÜNNER, José JOAQUín (2006): Curso: nuevos escenarios en la gestión pública. En: http://mt.educarchile.cl.

Celade (1988): Boletín Demográfico, Año 21, №41. Santiago: CELADE.

CEPAL, (2006): «La protección social de cara al futuro: acceso, financiamiento y solidaridad». En: www.eclac.cl.

Centro de Estudios del Trabajo Cetra/Ceal (1983): Irrupción del movimiento obrero en la vida nacional: período de exclusión, 1880-1920. En: www.memoriachilena.cl.

Charlín, MARCElo Y JÜRGEN Weller (editores) (2006): «Juventud y mercado laboral». Santiago: CEPAL, FLACSO y GTZ.

DuARTE, Claudio (2002): «Mundos jóvenes, mundos adultos: lo generacional y la reconstrucción de los puentes rotos en el liceo. Una mirada desde la convivencia escolar». Última Década $\mathrm{N}^{\circ} 16$. Viña del Mar: Ediciones CIDPA.

FeiXa, CARLeS (S/F): Antropología de las edades.

En: www.cholonautas.edu.pe.

(2005): «Los nuevos modelos culturales». Fin de Siglo Vol. 20. Lima: Salvat Editores.

GARCÍA, BRÍGIDA Y Olga Rojas (2002): «Cambio en la formación y disoluciones de las uniones en América Latina». Papeles de Población $\mathrm{N}^{0} 32$. Toluca: Universidad Autónoma del Estado de México.

GYSLING, JACQUELINE Y CRISTINA BENAVENTE (1996): «Trabajo, sexualidad y poder. Mujeres de Santiago». Nueva Serie FLACSO-Chile. Santiago: FLACSO.

GHIARDO, FeliPe (2004): «Generaciones y juventud: una relectura desde Mannheim y Ortega y Gasset». Ultima Década ํ⒛ Valparaíso: Ediciones CIDPA.

GÜELL, PEDRO (1999): «Familia y modernización en Chile».

En: www.desarrollohumano.cl.

— (coordinador) (1998): «Vivir la inseguridad: cotidianeidad y trayectorias de familia». Desarrollo humano en Chile. Las paradojas de la modernización. Santiago: PNUD.

GutiÉRREZ, EUGENIO y PATRICIO RÍOS (2006): «Envejecimiento y campo de la edad: elementos sobre la pertinencia del conocimiento gerontológico». Última Década №25. Valparaíso: Ediciones CIDPA. 
GuZMÁN, José y SANDRA HuENCHUAN (2004): «Políticas hacia las familias con adultos mayores: notas preliminares». En: Cambio de las familias en el marco de las transformaciones globales: necesidad de politicas públicas eficaces. Reunión de expertos, 28 a 29 octubre de 2004. Santiago: CEPAL.

HERNÁNDEZ, LuIS (1995): «Complejidades y desafíos actuales de la descentralización en un Estado unitario». En: Chile, ruta al tercer milenio. Santiago: Itesa.

HOPENHAYN, MARTín (2004): «Cambios en el paradigma del trabajo e impacto en la familia». En: Cambio de las familias en el marco de las transformaciones globales: necesidad de políticas públicas eficaces. Reunión de expertos, 28 a 29 octubre de 2004. Santiago: CEPAL.

INJUV: Encuestas nacionales de juventud (1994-1997-2000-2003-2006). En: www.injuv.gob.cl.

JELIN, ElizABETH (1994): «Las familias en América Latina». En: Isis Internacional. Familias siglo XXI. Ediciones de las mujeres №20. Santiago: Isis Internacional.

LECHNER, NORBERT (1999): «Desafíos de un desarrollo humano: individualización y capital social». Contribución al Foro Desarrollo y Cultura, organizado para la Asamblea General del Banco Interamericano del Desarrollo (BID), Paris, marzo de 1999. En: www.desarrollohumano.cl.

MANNHEIM, KARL (1990): Le problème des générations. Paris: Nathan.

Martín CRIADO, EnRIQUe (1998): Producir la juventud. Madrid: Istmo.

_ (S/F): «Generaciones/clases de edad». En: http://www.ucm.es.

MATTELART, MiCHELlE (1975): «La mujer y la línea de masa de la burguesía: el caso de Chile». La mujer en América Latina. México: Secretaría de Educación Pública.

MINISTERIO DE EDUCACIÓN (MINEDUC) (S/F): En: http://www.mineduc.cl.

MiNiSTERIO DE HACIENDA (2005): «Estado de la hacienda pública 2005, años de escolarización promedio de la población mayor de 15 años. Período 1850-1990». En: www.hacienda.cl.

Ministerio de PlanifiCACIÓn (MIDEPLAN) (2006): Encuesta CASEN. Santiago: Mideplan.

Montecino, Sonia (2007): Madres y huachos. Santiago: Catalonia.

OLAVARRÍA, JOSÉ (coordinador) (2004): Adolescentes: conversando la intimidad. Vida cotidiana, sexualidad y masculinidad. Santiago: FLACSO. (2005a): «Dónde está el nuevo padre? Trabajo doméstico: de la retórica a la práctica». En XIMENA VALDÉS y TERESA VALDÉS (editoras): Familia y vida privada. Santiago: FLACSO, CEDEM y UNFPA. (2005a) «Ser padre en Santiago de Chile». En: www.eurosur.org. (2005b) «Adolescentes/jóvenes: qué poco sabemos de ellos». En: Www.flacso.cl.

ORGANIZACIÓN MUNDIAL DE LA SALUD: En: http://www.sochog.cl. 
PARRINI, RoDRIGO (S/FA): «Apuntes acerca de los estudios de masculinidad. De la hegemonía a la pluralidad». En: www.eurosur.org.

(1999) «Los poderes del padre: paternidad e identidad masculina». Ponencia en Primer Encuentro de Estudios de Masculinidad, Santiago, 18 de noviembre. En: www.eurosur.org.

PALMA, IRMA (2000): «Transformaciones de la fecundidad en la sociedad chilena». En: www.pasa.cl.

PÉREZ DÍAZ, JULIO (2000): «Envejecimiento y esperanza de vida en salud». En: www.ced.uab.es.

Power, MARGARet (1997): «La Unidad Popular y la masculinidad». Revista La Ventana $\mathrm{N}^{\circ}$ 6. En: www.publicaciones.cucsh.udg.mx.

PNUD (1998): Segundo Informe del Desarrollo Humano en Chile. Santiago: PNUD.

— (2000): Tercer Informe de Desarrollo Humano en Chile. Más sociedad para gobernar el futuro. Santiago: PNUD.

RÍOS, PATRICIO: «El habitus de la edad». Documento inédito.

ROJAS, JORGE (2004): Moral y prácticas cívicas en los niños chilenos, 18801950. Santiago: Ariadna Ediciones.

Salazar, Gabriel y Julio Pinto (1999a): Historia contemporánea de Chile, Tomo I. Santiago: LOM ediciones.

$-\mathrm{y}-(1999 \mathrm{~b})$ : Historia contemporánea de Chile, Tomo II. Santiago: LOM ediciones.

$-\mathrm{y} \longrightarrow$ (2002a): Historia contemporánea de Chile, Tomo IV. Santiago: LOM ediciones.

$-\mathrm{y}-(2002 \mathrm{~b})$ : Historia contemporánea de Chile, Tomo V. Santiago: LOM ediciones.

SOCIEDAD ChILENA DE OBSTETRICIA Y GineCOlOGÍA (S/F): www.sochog.cl.

SCHKOLNIK, MARIANA (2004): «Tensión entre familia y trabajo». En: Cambio de las familias en el marco de las transformaciones globales: necesidad de políticas públicas eficaces. Reunión de expertos, 28 a 29 octubre de 2004. Santiago: CEPAL.

TIRONI, EUGENIO (2006): «El sueño chileno: comunidad, familia y nación». Santiago: Taurus. En: www.unifam.cl/documentos.

VALDÉS, XIMENA (2004): «Familias en Chile: rasgos históricos y significados actuales de los cambios». En: Cambio de las familias en el marco de las transformaciones globales: necesidad de políticas públicas eficaces. Reunión de expertos, 28 a 29 octubre de 2004. Santiago: CEPAL.

_ (S/F): «Lo privado y lo público: lugares de desigual disputa». Mesa Agenda Pro-género, Chile 21. En: www.fes.cl.

(2007): «Notas sobre la metamorfosis de la familia en Chile». Reunión de especialistas futuro de las familias y desafíos para las políticas públicas. 22-23 noviembre 2007, Santiago. En: www.eclac.org. 
VALDÉS, TERESA y ENRIQUe GOMARIZ (1992): Mujeres latinoamericanas en cifras. Santiago: Instituto de la Mujer.

— y José OlaVARIA (1998): «Ser hombre en Santiago de Chile: a pesar de todo, un mismo modelo». En Valdés y Olavarría (editores): Masculinidades y equidad de género en América Latina. Santiago: FLACSO y UNFPA.

—- JACQUELINE GYSLING y M. CRISTINA BENAVENTE (1999): El poder en la pareja, la sexualidad y la reproducción. Mujeres de Santiago. Santiago: FLACSO.

VidAL, ViRginia (1972): «La emancipación de la mujer». La Ventana $\mathrm{N}^{\circ} 6$. Santiago. 